\title{
Advanced Control Strategies for Direct-Drive PMSG Wind Turbine Systems: Direct Predictive Torque Control Approaches
}

\author{
Zhenbin Zhang, José Rodríguez, and Ralph Kennel
}

\begin{abstract}
Full power scale back-to-back power converter PMSG wind turbine system, with direct-drive configuration, is an attractive solution, particularly for off-shore wind energy applications. For such systems, (nonlinear) direct control, which requires neither a modulation process nor cascaded linear controllers, but will operate the system at very high control dynamics, is a very promising control class. In this work, we reviewed and experimentally assessed the classical (C-), the duty-optimal (DO-), the ripple-reduced (RR-) and the multi-vector direct model predictive torque control (MV-DMPTC) solutions to deal with the generator side control of grid-tied full power scale back-to-back power converter PMSG wind turbine systems. Their theoretical background, realizations and control performances are presented and discussed. The realizations and experimental assessments of all the discussed control approaches are carried out with a fully FPGA based realtime controller, at a lab-constructed test-bench. The resource usage and implementation complexity are provided. Comprehensive evaluation results are given at the end.
\end{abstract}

Index Terms-Direct torque control, FPGA, nonlinear control, predictive torque control, time optimal control, voltage source back-to-back power converters, wind turbine system with permanent magnet synchronous generator.

\section{INTRODUCTION}

$\mathrm{W}$ IND energy installations have steadily increased over the last years.Wind turbine systems (WTSs) using fullscale back-to-back power converter and permanent-magnet synchronous generator (PMSG) with direct-drive configuration (without mechanical gear) are an interesting and promising alternative to doubly-fed induction generator based WTSs, due to its higher power density and more degrees of freedom in control and during grid faults. The electrical block diagram of a WTS with direct-drive PMSG and grid-tied back-to-back converter is shown in Fig. 1. Such a configuration allows for (see, e.g., [1]): (i) bidirectional power flow, (ii) an operation over a wide wind speed range, (iii) small DC-link capacitor volume and size, (iv) simple

Manuscript received August 20, 2017. This work was supported by DFG funding under Grant Nr.: KE817/32-1.

Z. Zhang and R. Kennel are with the Institute for Electrical Drive Systems and Power Electronics, Technical University of Munich, Munich 80333, Germany (e-mail: zhenbin.zhang@tum.de; ralph.kennel@tum.de). J. Rodríguez is with Faculty of Engineering, Universidad Andres Bello, Santiago 8370146, Chile (e-mail: jose.rodriguez@unab.cl). (corresponding author: Zhenbin Zhang.

Digital Object Identifier 10.24295/CPSSTPEA.2017.00020

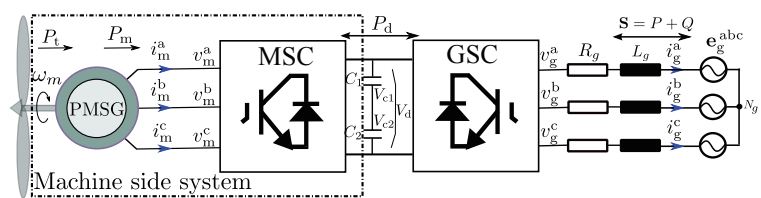

Fig. 1. A simplified structure of a grid-tied direct-drive back-to-back power converter PMSG wind turbine system, where $x_{\mathrm{g}, \mathrm{m}}$ are the variables for grid $(g)$ and machine $(m)$ side, $x \in\{i, e, v, R, L\}$ represents the current, grid and converter voltage (vector), resistance and inductance, respectively, $V_{\mathrm{d}}, \omega_{m}$, $P, Q$ are the DC-link voltage and rotor speed, grid side active and reactive power, respectively. $P_{t}$ and $P_{m}$ is the power output of the wind turbine and generator, respectively. "MSC" and "GSC" represent "machine side converter" and "grid side converter".

fault-ride through capabilities, and (v) reduced maintenance. These features make such WTSs attractive, in particular, for off-shore applications.

Control schemes for the machine side convertor (MSC) of such systems (as shown in Fig. 1) can be divided into two classes (see, e.g., [2], [3]): (i) (Linear) control schemes (with modulator) (e.g. with space vector modulation (SVM)), such as (a) PI controller methods, e.g. field-oriented control (FOC) or (b) direct torque control (DTC) with modulator, and (c) deadbeat-like model predictive control (DBC) methods; and (ii) (nonlinear) direct control schemes (without modulator) such as (a) DTC with switching table (ST-DTC) and (b) (nonlinear) direct model predictive control (DMPC) approaches. From the concept point of view, the first class (partially) approximates the plant (i.e., the power converters and drives) as a linear and continues system, thereby, applying the "timed-average principle" with a modulator to emulate certain continues commands to the system. However, a switching power converter-fed energy conversion system is in essence a nonlinear and switching-mode plant. Modern digital controllers process a control algorithm in discrete format as well. Therefore, a more proper control philosophy shall be nonlinear direct control, which requires no linear and continuous approximation, but takes the nonlinear and switching-mode nature of the power converters and digital controllers into account and combines the modulation and switching sequence selection processes into a single step.

Switching table based direct control (direct torque control (ST-DTC) [4], [5] for machine side, and direct power control (ST-DPC) for grid side), which was originally developed in the 1980s for induction motor drives, has already been a very matured concept. In such solutions, the switching se- 
quence are directly selected based on the mistake between the reference and the actual values with an offline designed switching table. No modulator or linear controller is required therein. Robust to parameter variations and very fast in control dynamics are their property. However, the switching table is inflexible (to deal with multiple control targets) and the control variable ripples highly depend on the sampling frequency of the system.

Recently, DMPC has been developed very fast in the field of power electronics and electrical drives. For such concept, instead of using a switching table, a very flexible cost function (also called the objective function) is utilized to define the control objectives and the full system model (including the power converter) is taken into account to determine an optimal control sequence. Similarly, no (complex) modulation process is required therein. Shortcoming, in the analogy to the ST-DTC approach, is that, the control variable ripples are very high in comparison with the classical modulator based solutions, due to that, only one switching vector will be selected and applied in a whole sampling interval.

To conquer this, many alternatives have been developed, e.g., the duty-optimal two-vector based direct predictive control (DO-DMPTC) method was proposed and presented in [6], [7], a ripple-reduced two vector direct model predictive torque control (RR-DMPTC) was proposed and reported in [8], [9], a multi-vector direct model predictive power control (MVDMPPC) was proposed and evaluated in [10], a long-horizon direct model predictive torque control (LHDMPTC) was investigated in [11], [12], etc. Although LHDMPTC results in a considerably improved control performances, in particular, at very low switching frequency cases, the required computational demands increase exponentially with the prediction horizon, and the key technologies to use such solution lie at the computationally intelligent methods to solve the so-called nonlinear mixed-integer optimization problem [12], [13], which goes beyond the scope of this work. On the other hand, the DO-, the RR-, and the MVDMPTC remain still within the short-horizon direct model predictive control domain, invoking the so-called time-optimal concept. Simple modifications from the C-DMPTC will result in considerably improved control performances. Their slightly higher computational demands can be easily coped with by using field programmable gate array (FPGA) based real-time controller targets, which have already been a very popular solution (see e.g., [14]).

In this work, the C-, the DO-, the RR- and the MV-DMPTC control approaches for the machine side control of a two voltage level full-scale back-to-back power converter based PMSG wind turbine system are reviewed and comprehensively assessed. Both the controller designs and their theoretical backgrounds are discussed in detail. All methods are implemented on an FPGA-based real-time platform. Their control performances are compared experimentally with a labconstructed grid-tied PMSG wind energy system emulator.

This paper is organized as follows: In Sec. II, the machine side part of a grid-tied direct-drive back-to-back power converter PMSG wind turbine system is described and modeled in both continues- and discrete-time formats. In Sec. III, after revisiting the $\mathrm{C}-$, the DO- and the RR-DMPTC solutions, we introduce a time-optimal MV-DMPTC approach. In Sec. $\mathrm{IV}$, the FPGA design and measurement results are presented. At the end, Sec. V concludes this paper.

\section{System Description And Modeling}

In this section machine side system of a grid-tied fullscale back-to-back power converter PMSG wind turbine is described and modeled in both continues- and discrete-time formats to ease the understanding of the following controller design and analysis sections.

\section{A. Continuous-Time Models}

The dynamics of a PMSG are given by [13]

$$
\left.\begin{array}{rl}
\boldsymbol{v}_{\mathrm{m}}^{\mathrm{dq}} & =R_{\mathrm{s}} \boldsymbol{i}_{\mathrm{m}}^{\mathrm{dq}}+\overbrace{\left[\begin{array}{cc}
c_{\mathrm{s}}^{\mathrm{d}} & 0 \\
0 & L_{\mathrm{s}}^{\mathrm{q}}
\end{array}\right] \frac{\mathrm{d}}{\mathrm{d} t} \boldsymbol{i}_{\mathrm{m}}^{\mathrm{dq}}+N_{\mathrm{p}} \omega_{m} \overbrace{\left[\begin{array}{cc}
0 & -1 \\
1 & 0
\end{array}\right]}^{=: \boldsymbol{\psi}_{\mathrm{s}}^{\mathrm{dq}}}}^{=: \boldsymbol{J}} \\
\boldsymbol{\psi}_{\mathrm{s}}^{\mathrm{dq}} & =\boldsymbol{L}_{\mathrm{s}}^{\mathrm{dq}} \boldsymbol{i}_{\mathrm{m}}^{\mathrm{dq}}+\boldsymbol{\psi}_{\mathrm{pm}}^{\mathrm{dq}} \\
\frac{\mathrm{d}}{\mathrm{d} t} \omega_{m} & =\frac{1}{\Theta}[T_{l}-\underbrace{\left(N_{\mathrm{p}}\left(\boldsymbol{i}_{\mathrm{m}}^{\mathrm{dq}}\right)^{\top} \boldsymbol{J} \boldsymbol{\psi}_{\mathrm{s}}^{\mathrm{dq}}\right)}_{=: T_{e}}]
\end{array}\right\}
$$

where $v_{\mathrm{m}}^{\mathrm{dq}}=\left(v_{\mathrm{m}}^{\mathrm{d}}, v_{\mathrm{m}}^{\mathrm{q}}\right)^{\top}, i_{\mathrm{m}}^{\mathrm{dq}}=\left(i_{\mathrm{m}}^{\mathrm{d}}, i_{\mathrm{m}}^{\mathrm{q}}\right)^{\top}, R_{\mathrm{s}}, L_{\mathrm{s}}^{\mathrm{d}}, L_{\mathrm{s}}^{\mathrm{q}}, N_{\mathrm{p}}, \omega_{\mathrm{m}}, \psi_{\mathrm{pm}}^{\mathrm{dq}}$ $=\left(\psi_{\mathrm{pm}}, 0\right)^{\top}, \Theta, T_{\mathrm{e}}$ and $T_{l}$ are machine (applied) voltage and current vector, stator resistance, $d$-, $q$-stator inductances, number of pole pairs, machine angular velocity, permanent magnet flux linkage, inertia, electromagnetic torque and load torque, respectively. For two-level power converters, the admissible switching state $u_{\mathrm{i}}$ is within a finite set (See Fig. 2(a)), i.e.,

$\boldsymbol{u}_{\mathrm{i}} \in \mathcal{U}:=\left\{\boldsymbol{u}_{0}(000), \boldsymbol{u}_{1}(001), \cdots, \boldsymbol{u}_{6}(101), \boldsymbol{u}_{7}(111)\right\}$

Neglecting losses, the converter voltage vector in the $d q$-reference frame is calculated as [15]

$$
\boldsymbol{v}_{\mathrm{m}}^{\mathrm{dq}}\left(\boldsymbol{u}_{\mathrm{i}}\right)=\mathbf{T}_{\mathrm{P}} \cdot \mathbf{T}_{\mathrm{C}} \cdot \underbrace{\frac{V_{\mathrm{d}}}{3}\left[\begin{array}{ccc}
2 & -1 & -1 \\
-1 & 2 & -1 \\
-1 & -1 & 2
\end{array}\right]}_{=: \boldsymbol{T}_{\mathrm{SW}}} \boldsymbol{u}_{\mathrm{i}}
$$

where $T_{P}$ and $T_{C}$ are the Park and Clark transformation matrices (See e.g., [13]), $\mathrm{V}_{\mathrm{d}}$ is the DC-link voltage.

\section{B. Discrete-Time Models}

Defining the slope of $x(t)$ at sampling instant $\mathrm{k}$ as $g_{x}(k)=$ $\left.\frac{\mathrm{d} x(t)}{\mathrm{d} t}\right|_{t=\mathrm{k} \cdot \mathrm{T}_{\mathrm{s}}}$, where $T_{\mathrm{s}}$ is the sampling interval, and applying the Euler-Forward equation (i.e, $\frac{\mathrm{d} \vec{x}(t)}{\mathrm{d} t} \approx \frac{\vec{x}_{[\mathrm{k}+1]}-\vec{x}_{[\mathrm{k}]}}{T_{\mathrm{s}}}$ ) to (1), 
yields the discrete-time slopes of the currents and electron-magnetic torque of the generator, at the drive force of switching vector $u_{\mathrm{i}}$, as [13]

$$
\begin{aligned}
g_{i_{\mathrm{m}}^{\mathrm{d}}}^{\boldsymbol{u}_{\mathrm{i}}}(k)=\left.\frac{\mathrm{d} i_{\mathrm{m}}^{\mathrm{d}}(t)}{\mathrm{d} t}\right|_{t=\mathrm{k} \cdot \mathrm{T}_{\mathrm{s}}} & =\frac{R_{\mathrm{s}}}{L_{\mathrm{s}}} i_{\mathrm{m}}^{\mathrm{d}}(k)+N_{\mathrm{p}} \omega_{\mathrm{m}}(k) i_{\mathrm{m}}^{\mathrm{q}}(k) \\
& +\frac{v_{\mathrm{m}}^{\mathrm{d}, u_{\mathrm{i}}}(k)}{L_{\mathrm{s}}} \\
g_{i_{\mathrm{m}}^{\mathrm{q}}}^{\boldsymbol{u}_{\mathrm{i}}}(k)=\left.\frac{\mathrm{d} i_{\mathrm{m}}^{\mathrm{q}}(t)}{\mathrm{d} t}\right|_{t=\mathrm{k} \cdot \mathrm{T}_{\mathrm{s}}} & =\frac{-R_{\mathrm{s}}}{L_{\mathrm{s}}} i_{\mathrm{m}}^{\mathrm{q}}(k)-N_{\mathrm{p}} \omega_{\mathrm{m}}(k) i_{\mathrm{m}}^{\mathrm{d}}(k) \\
& -N_{\mathrm{p}} \omega_{\mathrm{m}}(k) \frac{\psi_{\mathrm{pm}}}{L_{\mathrm{s}}}+\frac{v_{\mathrm{m}}^{\mathrm{q}, u_{\mathrm{i}}}(k)}{L_{\mathrm{s}}}
\end{aligned}
$$

The discrete models are useful to predict the future behaviors of the system, e.g., at $k+1$, at a drive force with vector $u_{\mathrm{i}}$,

$$
x(k+1)=x(k)+T_{\mathrm{s}} \cdot g_{\mathbf{x}}^{u_{\mathrm{i}}}(k),
$$

where $x \in\left\{i_{\mathrm{m}}^{\mathrm{d}}, i_{\mathrm{m}}^{\mathrm{q}}, T_{\mathrm{e}}\right\}$.

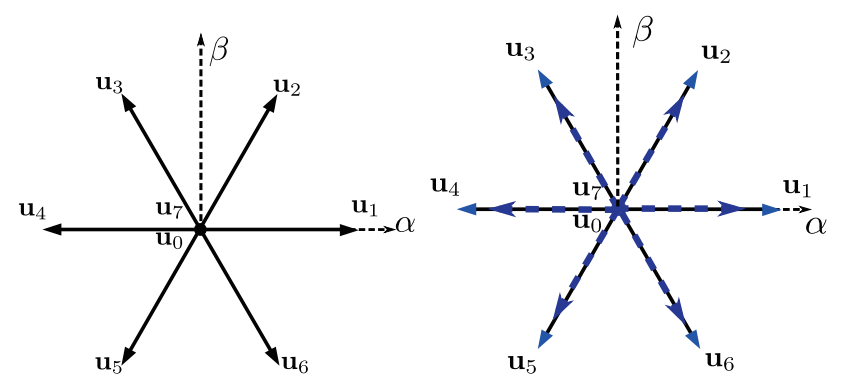

(a) C-DMPTC (also the original

(b) DO-DMPTC available voltage vectors)

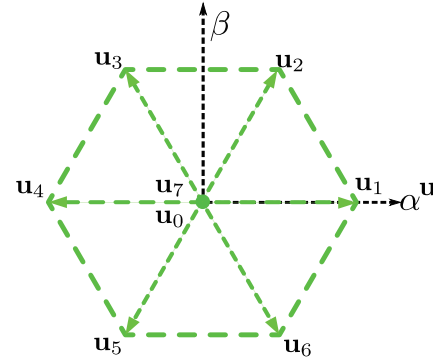

(c) RR-DMPTC

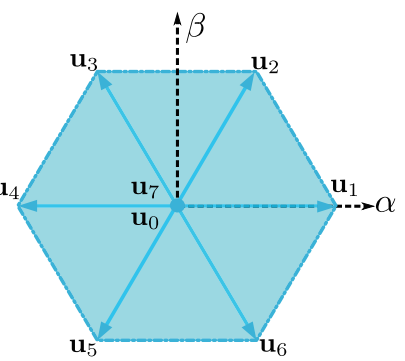

(d) MV-DMPTC
Fig. 2. Candidate switching vector ranges/planes for the C-, the DO-, the RR- and the MV-DMPTC solutions.

\section{Classical and Advanced Direct Predictive Torque Control Methods}

For a grid-tied direct-drive PMSG wind turbine system, the in-

${ }^{1}$ In this work a surface-mounted PMSG was used, i.e., $L_{s}^{d} \approx L_{s}^{q}$. Therefore, $i_{\mathrm{m}}^{\mathrm{d}^{\mathrm{m}}}:=0$ will lead to the so-called MTPA control, for which, the detailed analysis can be found in e.g., [13].

${ }^{2}$ Note that, for both the DO- and RR-DMPTC methods to be introduced in the following, $\gamma_{S}$ in (6) is set to be zero (i.e., the constraint is not considered) so that the cost function becomes (globally) differentiable, and the duty cycles can be calculated. ner loop control objectives of the machine (here the PMSG) side includes: $\left(\mathrm{Co}_{1}\right)$ torque tracking with fast dynamics and accuracy (to meeting the outer maximum power point tracking), and $\left(\mathrm{Co}_{2}\right)$ to achieve maximum efficiency and to best utilize the available stator currents, i.e., to meet the socalled maximum-torque-per-ampere (MTPA) requirements, and $\left(\mathrm{CO}_{3}\right)$ the system shall operate within the allowed power range/constraint. Additionally, low torque/current ripples are desirable to reduce losses and mechanical bearing. Therefore a cost function of

$J\left(\boldsymbol{u}_{\mathrm{i}}\right)=\sum_{h=k}^{k+N-1}(\underbrace{\left(T_{\mathrm{e}}^{*}-T_{\mathrm{e}}(k+1)\left(\boldsymbol{u}_{\mathrm{i}}\right)\right)^{2}+\gamma_{\mathrm{i}_{\mathrm{m}}}\left(i_{\mathrm{m}}^{\mathrm{d}}(k+1)\left(\boldsymbol{u}_{\mathrm{i}}\right)\right)^{2}}_{=: J_{\mathbf{T S}}}+J_{\mathbf{C S}})$,

can be defined for a surface-mounted $\mathrm{PMSG}^{1}$ to guarantee the above mentioned control objectives, where $h \in \mathbb{N}^{+}$is the prediction horizon, $i \in\{0,1, \ldots, 6,7\}$ represents the number of the available switching vectors, $J_{\mathrm{TS}}$ represents the targets for torque and MTPA control.

$$
J_{\mathbf{C S}}=\gamma_{\mathrm{I}} \cdot \text { if }\left\{\left\|\boldsymbol{i}_{\mathrm{m}}^{\mathrm{dq}}(k+1)\right\|>\left\|\boldsymbol{I}_{\mathrm{m}}\right\|^{\max }\right\}
$$

represents the limitation constraint of the system, where $\left\|I_{m}\right\|^{\max }$ is the system permissible current limit, $\gamma_{\mathrm{I}}(>0)$ is the weighting factor ${ }^{2}$. In this work, a "one-step" prediction is considered for a fair comparison.

In the following, within the nonlinear direct control class, the classical direct model predictive torque control (i.e., the C-DMPTC), and three recently reported more advanced direct model predictive torque control solutions (i.e., the DO-, the RR- and the MV-DMPTC) are presented. Note that, the outer speed controller (here the same proportional integration (PI) controller as in [16], [17] is adopted) is not the scope of this paper and is therefore not redundantly reported.

\section{A. Classical DMPTC (C-DMPTC)}

The C-DMPTC scheme [13] evaluates the given cost function (6) for all the admissible (finite) set $\mathcal{U}$ (see (2)) by using the prediction model presented in (5), i.e.,

$\left(\boldsymbol{u}_{\mathrm{x}}^{\star}, \boldsymbol{t}_{\mathrm{x}}^{\star}\right):=\arg \min _{x \in\{0, \ldots, 7\}} J\left(\boldsymbol{u}_{\mathrm{i}}\right)\left(\boldsymbol{i}_{\mathrm{m}}^{\mathrm{dq}}(k+1), T_{\mathrm{e}}(k+1)\right)$,

However, the optimal vector $u_{\mathrm{x}}^{\star}$ in this case can only be one of the original eight fundamental vectors, i.e., one of the black lines or the origin in Fig. 2(a), and will be applied for a whole control interval (so $t_{\mathrm{x}}^{\star}:=T_{\mathrm{s}}$ ), i.e., "one-vector-per-control-interval". Therefore, when the ideal equivalent voltage vector (which could "zerolize" the difference between the reference and the real value) is far away from these fundamental vectors, a rough approximation will lead to big control variable ripples during the steady state. Inspired by this, a duty-opt direct model predictive control method (i.e., the DO-DMPTC) was firstly proposed in [6], [7]. In the following, it will be detailed. 


\section{B. Duty-Optimal DMPTC (DO-DMPTC)}

In the concept of the DO-DMPTC, instead of "one-vector-per-control-interval", two vectors, i.e., one active and one zero vector, are chosen to minimize the cost-function. Detailed realizations are as follows: Inserting (5) into the targeting set of (6) and invoking the time-optimal concept [10], [14], i.e.,

$$
\begin{aligned}
& \frac{\mathrm{d} J_{\mathbf{T S}}\left(\boldsymbol{i}_{\mathrm{m}}^{\mathrm{dq}}(k+1), T_{\mathrm{e}}(k+1),\left(\boldsymbol{u}_{\mathrm{x}}^{\star}, \boldsymbol{u}_{\mathrm{y}}^{\star}\right)\right)}{\mathrm{d} \boldsymbol{t}_{\mathrm{x}}^{\star}} \stackrel{!}{=} 0, \\
& \text { s.t. : } \quad \boldsymbol{t}_{\mathrm{x}}^{\star}+\boldsymbol{t}_{\mathrm{y}}^{\star}=T_{\mathrm{s}}
\end{aligned}
$$

for all the six neighboring vector pairs ${ }^{3}$. Then through the following optimization process of

$$
\begin{aligned}
& \quad\left(\boldsymbol{u}_{\mathrm{x}}^{\star}, \boldsymbol{t}_{\mathrm{x}}^{\star}, \boldsymbol{u}_{\mathrm{y}}^{\star}, \boldsymbol{t}_{\mathrm{y}}^{\star}\right):= \\
& \arg \min _{x \in\{1, \cdots, 6\}, y \in\{0,7\}} J_{\mathbf{T S}}\left(\boldsymbol{i}_{\mathrm{m}}^{\mathrm{dq}}(k+1), T_{\mathrm{e}}(k+1)\right),
\end{aligned}
$$

The final solutions of $u_{\mathrm{x}}^{\star}, t_{\mathrm{x}}^{\star}, u_{\mathrm{y}}^{\star}$ and $t_{\mathrm{y}}^{\star}$ will be obtained. In the up coming interval, the switching vectors of $u_{\mathrm{x}}^{\star}$ and $u_{\mathrm{y}}^{\star}$ will be applied with their time durations of $t_{x}^{\star}, t_{y}^{\star}$, respectively. Such process in essence results in that, an equivalent vector of $u^{\mathrm{DO}}$, in phase with the selected active vector, with an optimized length can be synthesized, i.e., the available candidate switching vectors have been greatly extended to arbitrary lengths at the phases of all the original active switching vectors (See Fig. 2(b)). Therefore, better steady state performance than the C-DMPTC can be achieved. However, an equivalent vector with phases (directions) other than that of the fundamental active vector cannot be synthesized; the performance improvement is hence still limited.

\section{Ripple-Reduced DMPTC (RR-DMPTC)}

Inspired by the analysis above, a so-called "ripple reduced direct model predictive control" (RR-DMPC) scheme was proposed to further enhance the system performance [8], [13]. Different from both the C- and the DO-DMPTC schemes, with the RR-DMPC, an equivalent vector, is still synthesized with two vectors. However, instead of only one active and one zero vector, any of the neighboring vector pairs, including the two active ones are also used. To realize RR-DMPTC concept, again, the time-optimal concept will be applied, considering both the vector pairs of two active ones, and one active with one zero vectors. In the analogy, through the following optimization process of

$$
\begin{aligned}
& \left(\boldsymbol{u}_{\mathrm{x}}^{\star}, \boldsymbol{t}_{\mathrm{x}}^{\star}, \boldsymbol{u}_{\mathrm{y}}^{\star}, \boldsymbol{t}_{\mathrm{y}}^{\star}\right):= \\
& \arg \min _{x \in\{1, \cdots, 6\}, y \in\{0, \cdots, 7\}} J_{\mathbf{T S}}\left(\boldsymbol{i}_{\mathrm{m}}^{\mathrm{dq}}(k+1), T_{\mathrm{e}}(k+1)\right),
\end{aligned}
$$

\footnotetext{
${ }^{3}$ Note that, for all the DO-, RR- and MV-DMPTC solutions, $u_{\mathrm{x}}, u_{\mathrm{y}}$ are geometrical neighbors, following the relationship shown in Fig. 2(a), e.g., if $u_{\mathrm{x}}$ is (000), then $u_{\mathrm{y}}$ will be (001), if $u_{\mathrm{x}}$ is (001), $u_{\mathrm{y}}$ will be (011). This way will reduce both the computational efforts and switching transitions.
}

The optimal switching vectors with their operating time durations will be obtained and applied. Note that, $u_{\mathrm{y}}$ in this case includes also the active neighbors of $u_{\mathrm{x}}$. With such process, both optimized "phase" (at a full length, reaching the boundary of the hexagon plane) and an arbitrary length (at all the original active vector phases) are now available (see Fig. 2(c)). Easy to understand, better performances than both the C-DMPTC and DO-DMPTC are expected.

\section{Multiple-Vector DMPTC (MV-DMPTC)}

Although both the DO- and RR-DMPTC have extended the available candidate vector range, the entire potential of a power converters operation range, i.e, the whole hexagon plane, however, remains to be fully explored. Meanwhile, the undifferentiable constraint (e.g., system operation limits) was not included into the cost function. In [10], [13], a multiple vector direct model predictive power control (MV-DMPPC) scheme, which will utilize maximally three vectors, was presented to deal with the grid side control of the wind energy systems. Such scheme has fully utilized the whole plane of the hexagon, but still combines the optimization and modulation stages within one single process. The concept, when applied to the machine side with torque control, i.e., MV-DMPTC, will go through the following three steps:

1) Optimal direction detection: Ease to understand, only grouping an active pair will synthesize a new vector with different directions (other than any of the original active pair). In this step, all the neighboring active vector pairs will be selected. Again the time-optimal concept will be used to obtain their duration times. I.e., applying (14) for all the six neighboring vector pairs, and invoking again the minimization process of

$$
\begin{aligned}
& \left(\boldsymbol{u}_{\mathrm{x}}^{\star}, \boldsymbol{t}_{\mathrm{x}}^{\star}, \boldsymbol{u}_{\mathrm{y}}^{\star}, \boldsymbol{t}_{\mathrm{y}}^{\star}\right):= \\
& \arg \min _{x \in\{1, \ldots, 6\}, y \in\{1, \ldots, 6\}} J_{\mathbf{T S}}\left(\boldsymbol{i}_{\mathrm{m}}^{\mathrm{dq}}(k+1), T_{\mathrm{e}}(k+1)\right),
\end{aligned}
$$

will lead to a single optimal pair of vectors, with which, through the following equation of

$$
\boldsymbol{u}_{\text {new }}^{\star}:=\frac{\boldsymbol{t}_{\mathrm{x}}^{\star}}{T_{s}} \cdot \boldsymbol{u}_{\mathrm{x}}^{\star}+\frac{\boldsymbol{t}_{\mathrm{y}}^{\star}}{T_{s}} \cdot \boldsymbol{u}_{\mathrm{y}}^{\star} .
$$

a vector with a new direction will be obtained. This vector will be used in the following step to further tune its length, in combination with a zero vector.

2) Optimal vector length detection: Less ideally, the length of the above obtained new vector of $u_{\text {new }}^{\star}$ shall be tuned again, so an optimal vector can be obtain to achieve good steady state control performances. In this case, a zero vector shall be combined again with $u_{\text {new }}^{\star}$. This process requires again the time optimal concept, i.e.,

$$
\begin{aligned}
& \frac{\mathrm{d} J_{\mathbf{T S}}\left(\boldsymbol{i}_{\mathrm{m}}^{\mathrm{dq}}(k+1), T_{\mathrm{e}}(k+1),\left(\boldsymbol{u}_{\text {new }}^{\star}, \boldsymbol{u}_{\text {null }}^{\star}\right)\right)}{\mathrm{d} \boldsymbol{t}_{\text {new }}^{\star}} \stackrel{!}{=} 0, \\
& \text { s.t. : } \quad \boldsymbol{t}_{\text {new }}^{\star}+\boldsymbol{t}_{\text {null }}^{\star}=T_{\mathrm{s}}, \text { null } \in\{0,7\} .
\end{aligned}
$$


At the end, the final duration times of the above vectors $\left(u_{\mathrm{x}}, u_{\mathrm{y}}, u_{\text {null }}\right)$ will be obtained by

$$
\begin{aligned}
\boldsymbol{t}_{\mathrm{x}}^{\mathrm{opt}} & =m \cdot \boldsymbol{t}_{\mathrm{x}}^{\star}, \\
\boldsymbol{t}_{\mathrm{y}}^{\mathrm{opt}} & =m \cdot \boldsymbol{t}_{\mathrm{y}}^{\star}, \\
\boldsymbol{t}_{\mathrm{null}}^{\star} & =T_{\mathrm{s}}-\boldsymbol{t}_{\mathrm{x}}^{\mathrm{opt}}-\boldsymbol{t}_{\mathrm{y}}^{\mathrm{opt}},
\end{aligned}
$$

where $m:=\frac{\boldsymbol{t}_{\text {new }}^{\star}}{T_{\mathrm{s}}} \in[0,1]$.

3) Constraint inclusion: Not difficult to understand, the constraint violence happens when the current limit has already been reached but the reference tracking is still not met. A deep analysis for this situation will yield that, since the length of the vector obtained after Step D-1) can be tuned down to zero in Step D-2), only an improper direction/phase (i.e., direction optimization process in Step D-1) is improper) will lead to such violence. Inspired by this, a solution to include the system constraint by adding a comparison step, invoking the predictive constraint term in (6), after the aforementioned two steps, so to respect the whole cost function more properly.

\section{FPGA DESIGN}

The use of Field Programmable Gate Arrays (FPGAs) as part of the control platform in power electronics and electrical drive systems has been reported in both academic and industrial applications [18], [19]. In this work, all the algorithms (including C-, the DO-, the RR- and the MVDMPTC) are divided into sub-routines and implemented invoking the Single-Cycle-Timed-Loop (SCTL) technique on an entirely FPGA based platform. Due to the limited space, only the overall FPGA design structure for the MV-DMPTC is given in Fig. 3.

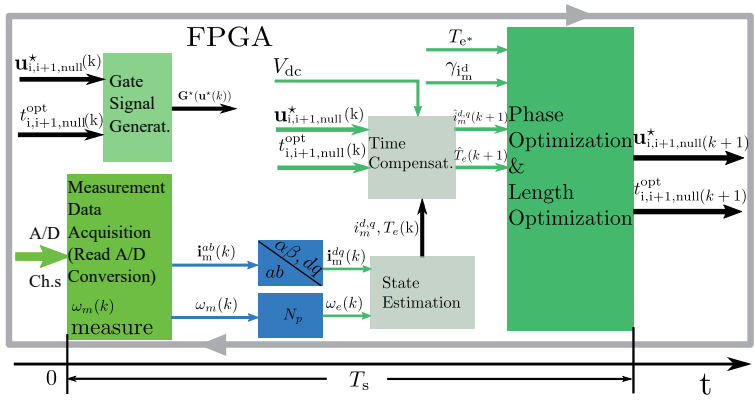

Fig. 3. FPGA design of the proposed MV-DMPTC control scheme (note that, to keep the scope, the speed and MPPT control loops are not presented in this figure. The time compensation part is the same as presented in e.g., [8], [10]).

\footnotetext{
${ }^{4}$ The top clock is $40 \mathrm{MHz}$, therefore, 1 [tick] $=1 / 40000000[\mathrm{~s}]$

${ }^{5}$ These data show the FPGA resource usage of the four schemes (solely for the grid side control). The resource cost for commutation interfaces, data acquisitions, signal saving blocks, etc. was not taken into account. Primary code optimizations are considered to save some resources, in particular for the last three methods (i.e., the DO-, RR- and MV-DMPTC), during their FPGA realizations.
}

The overall comparison during the FPGA program implementation is presented in TABLE I. As can be seen, higher resource usage is seen with the more advanced direct control approaches.

TABLE I

FPGA Design Overall Comparison

\begin{tabular}{lcc}
\hline \hline & Calculation Time $^{4}$ & Resource Usage $^{5}$ \\
\hline C-DMPTC: & $169[$ ticks] & $19 \%$ \\
DO-DMPTC: & $217[$ ticks] & $28 \%$ \\
RR-DMPTC: & $278[$ ticks] & $32 \%$ \\
MV-DMPTC: & $524[$ ticks] & $38 \%$ \\
\hline \hline
\end{tabular}

\section{EfFectiveness Evaluation and Analysis}

In this section the effectiveness evaluations of all the aforementioned approaches were compared with both simulation and experimental data. The system configuration and parameters are collected in TABLE II.

TABLE II

System PARAmeters

\begin{tabular}{ll}
\hline \hline Parameters & Values \\
\hline Generator stator inductance $\mathrm{L}_{\mathrm{s}}[\mathrm{H}]$ & $8 \cdot 10^{-3}$ \\
Generator stator resistance $\mathrm{R}_{\mathrm{s}}[\mathrm{Ohm}]$ & 1.3 \\
Generator nominal torque $/$ current $\mathrm{T}_{\mathrm{e}}^{\mathrm{n}} / \mathrm{I}_{\mathrm{m}}^{\mathrm{n}}[\mathrm{Nm} / \mathrm{A}]$ & $7.5 / 5$ \\
Permanent-magnet flux $\psi_{\mathrm{pm}}[\mathrm{Wb}]$ & 0.41 \\
Generator pole pair number $\mathrm{N}_{\mathrm{p}}[1]$ & 3 \\
Grid (phase) voltage $e_{\mathrm{g}}^{\mathrm{a}}[\mathrm{V}]($ peak) & 120 \\
Grid frequency $\omega_{\mathrm{g}}[\mathrm{rad} / \mathrm{s}]$ & $100 \pi$ \\
Grid side resistance $\mathrm{R}_{\mathrm{g}}[\mathrm{Ohm}]$ & $1.56 \cdot 10^{-3}$ \\
Grid side inductance $\mathrm{L}_{\mathrm{g}}[\mathrm{H}]$ & $16 \cdot 10^{-3}$ \\
DC-link capacitance $C:=C_{1}+C_{2}[\mu \mathrm{F}]$ & 1100 \\
\hline \hline
\end{tabular}

\section{A. Simulation Verification}

The overall control performance comparison among the C-, the DO-, the RR- and the MV-DMPTC methods are carried out through Matlab/Simulink as a preliminary concept of proof. The simulation results are shown in Fig. 4, where the same outer control loop and test situations are configured for all these four methods for a fair comparison. As can be seen, control dynamics remain almost the same, while greatly reduced steady state control variable ripples are seen with all the DO-, the RR- and the MV-DMPTC control solutions, with the MV-DMPTC being the best solution among the afore-discussed approaches.

To illustrate the detailed steady state control performances and to better understand the background forces for the steady state current/torque performances, a steady state control performances are shown in Fig. 5. As can be seen, at the same sampling frequency, the best current quality is achieved with the MV-DMPTC solutions, where the estimated synthesized voltage vector of $\hat{v}_{m}^{*}$ (estimated with the switching vector) 
shows the most smooth waveform. These voltage vectors at a large extend determines the steady state performances of a switching power converter driven system.

\section{B. Experimental Verification}

To experimentally evaluate the proposed scheme, a Lab-

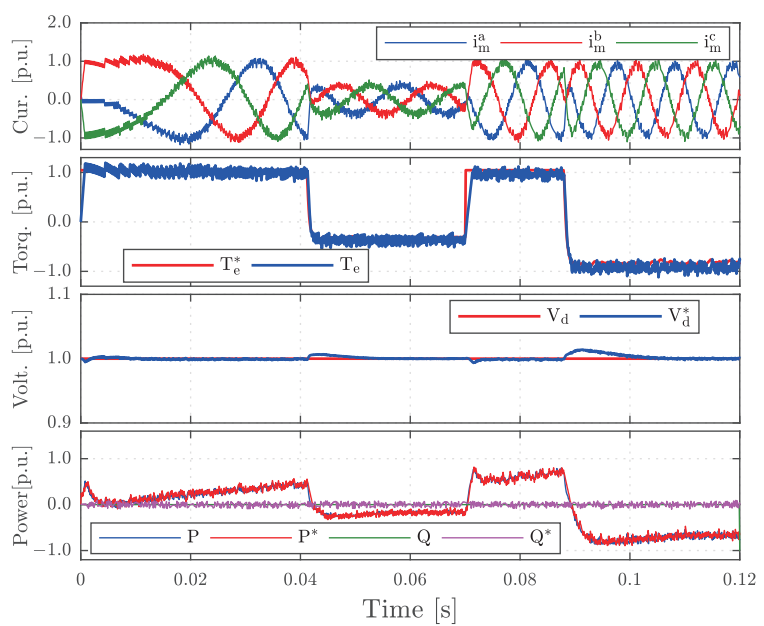

(a) C-DMPTC

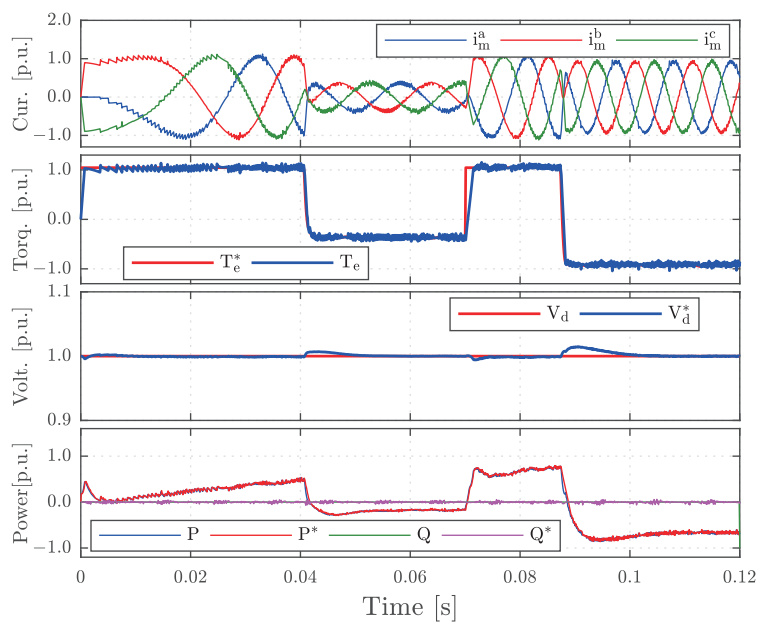

(b) DO-DMPTC

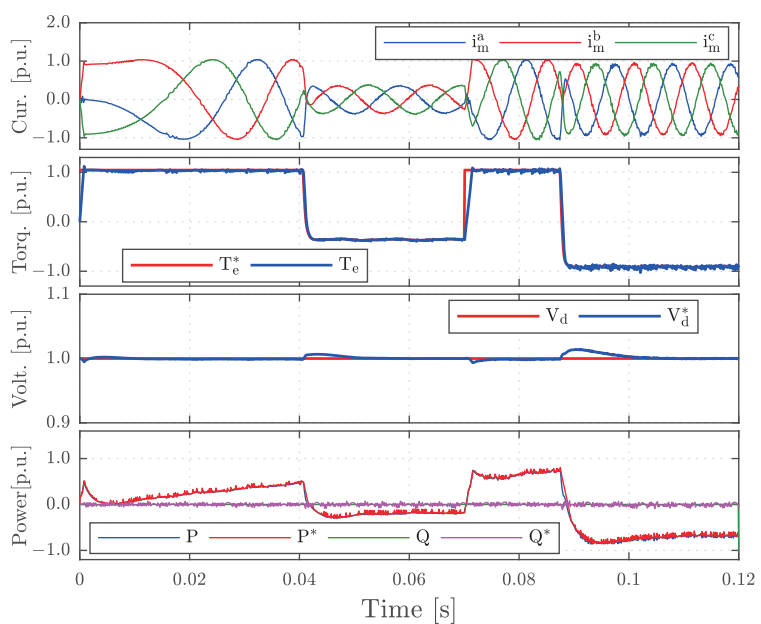

(c) RR-DMPTC

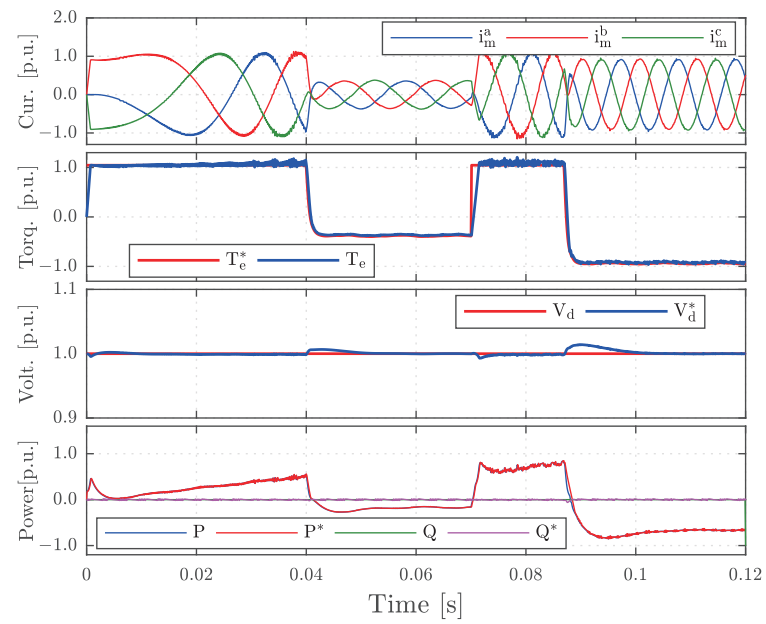

(d) MV-DMPC

Fig. 4. [Simulation data:] Overall control performance comparison. For each figure, from up to down are: the stator currents, electromagnetic torque, DC-link voltage, grid side power, grid side phase voltage and current, respectively, all in p.u. values.

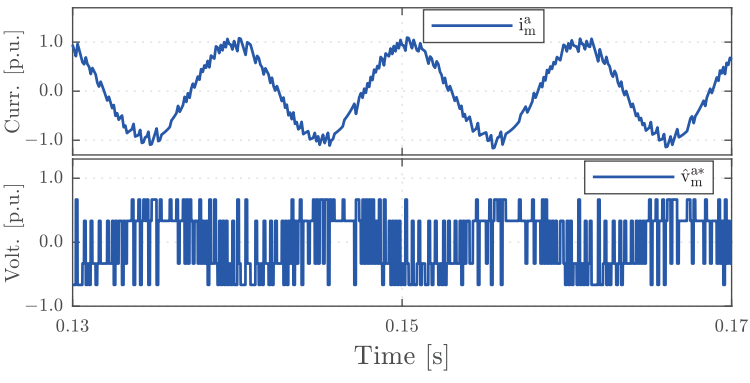

(a) C-DMPTC

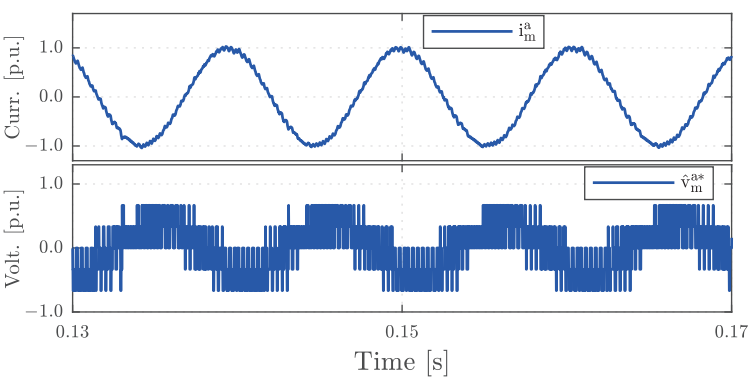

(b) DO-DMPTC

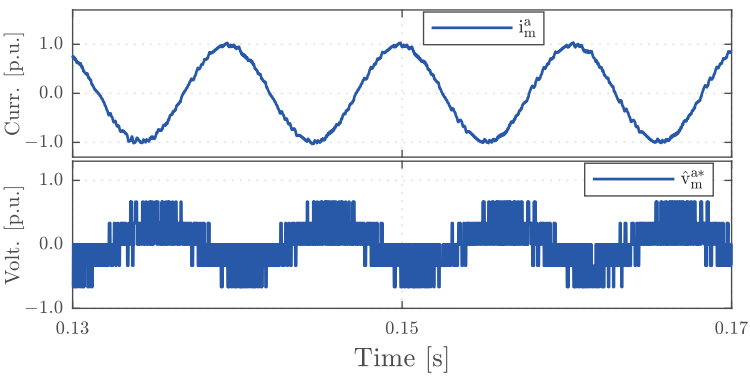

(c) RR-DMPTC

Fig. 5. [Simulation data:] Steady state control performances. For each figure, from up to down are: the generator stator currents and generator side converter (estimated) commanded voltages; grid side current and grid side converter (estimated) voltage, respectively. 


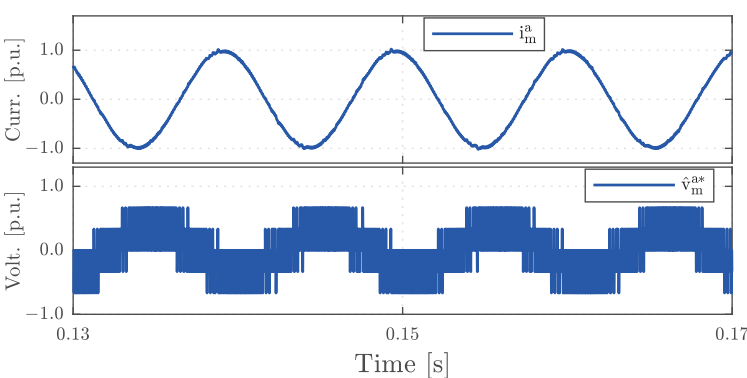

(d) MV-DMPTC

Fig. 5. (Continued.) [Simulation data:] Steady state control performances. For each figure, from up to down are: the generator stator currents and generator side converter (estimated) commanded voltages; grid side current and grid side converter (estimated) voltage, respectively.

prototype of a grid-connected PMSG wind turbine system with full scale back-to-back power converter and grid side (R)L-filter has been constructed. The laboratory prototype is depicted in Fig. 6. A variac is installed between grid and choke (RL-filter) to step down the grid side voltage for safety reasons. A fully FPGA based reconfigurable real-time system (NI-cRIO system) is used to implement all the discussed predictive controllers. The measurement results are shown in Fig. 7. As expected, considerably improved performances, in terms of smaller torque and current ripples and THD values, are seen in particularly with the RR- and MV-DMPTC solutions.

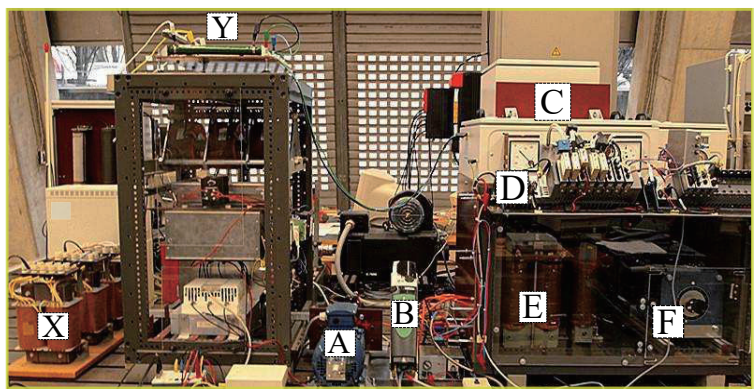

Fig. 6. Laboratory setup of a grid-connected back-to-back converter with $P M S G$ with (A) turbine emulator (AC-Motor) and PMSG, (B) load motor drive, $(\mathrm{C})$ back-to-back voltage source converter (constructed using Infineon IGBT modules), (D) NI-cRIO FPGA based controller, (E) grid-side choke (inductance $L_{g}$ with resistance $R_{g}$ ) and (F) grid-side variac, (X) and (Y) are the passive load for testing in islanding mode.

\section{Discussion}

In deep view of any control method for a switching power con-verter based system (including both the modulator and non-modulator based techniques) will yield such a conclusion: for all the available methods, forces to fulfill the control objectives are no more than three types, i.e., the admissible vector slopes during one control interval, duration time of each slope and their actuating arrangement (i.e., the pulse pattern). Considering only the first two, we can category the four direct model predictive control methods discussed in this paper with TABLE III.
As can be seen from TABLE III, both the vector slopes and their actuating times can be optimized with the MVDMPTC solution, therefore, best control performances are expected using such solution, in comparison with the $\mathrm{C}$-, the DO- and the RR-DMPTC methods.
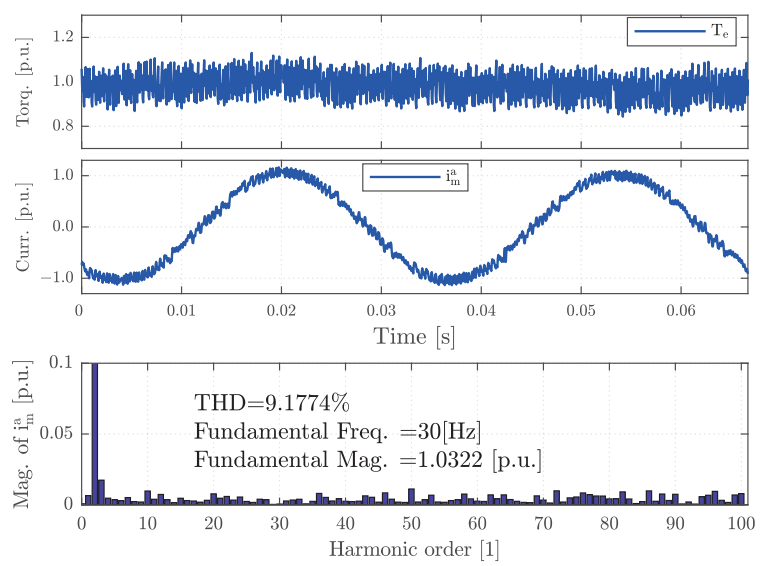

(a) C-DMPTC performances
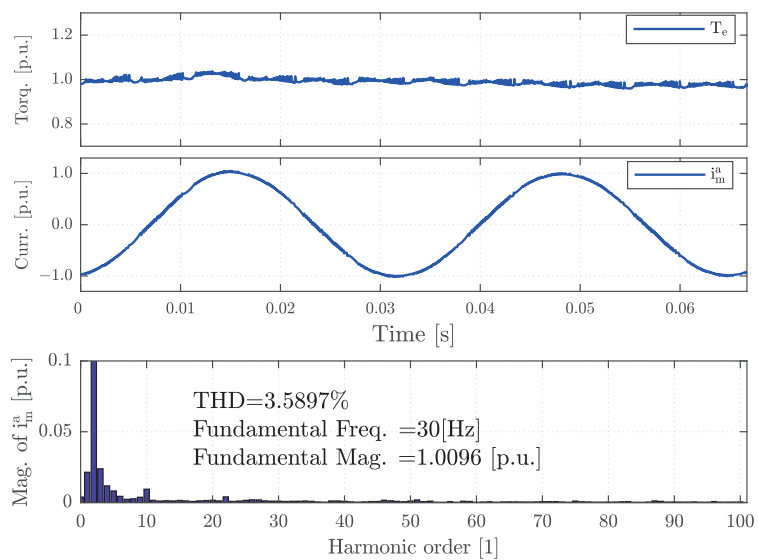

(b) DO-DMPTC performances
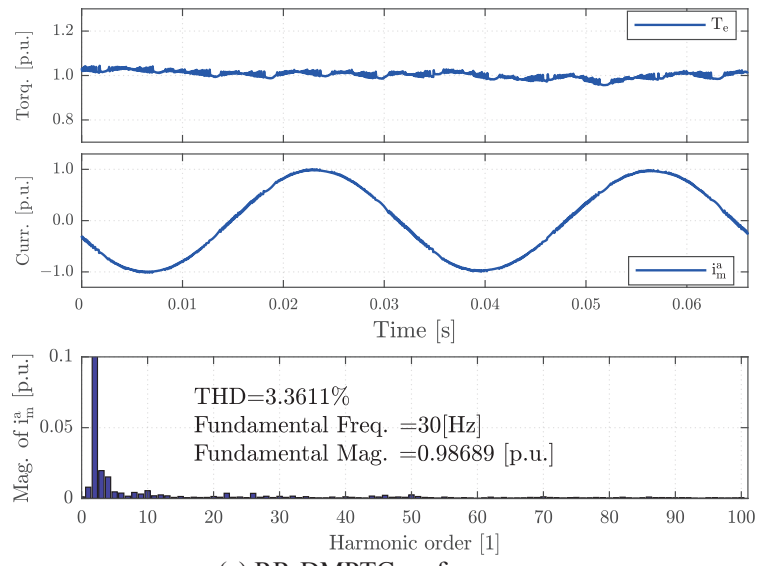

(c) RR-DMPTC performances

Fig. 7. [Experimental results:] Performances of the C-, the DO-, the RRand the MV-DMPTC methods. From up to down are the machine electromagnetic torque, the stator phase current and the current spectrum, respectively. 

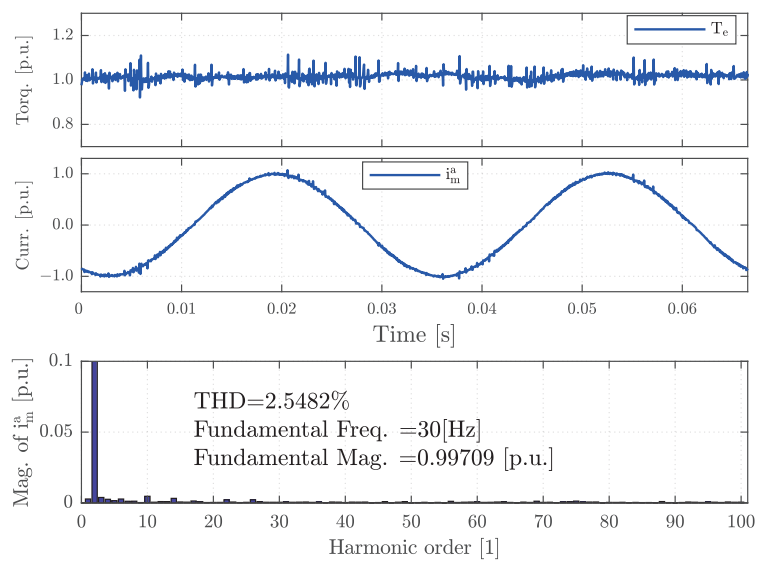

(d) MV-DMPTC performances

Fig. 7. (Continued.) [Experimental results:] Performances of the C-, the DO-, the RR- and the MV-DMPTC methods. From up to down are the machine electromagnetic torque, the stator phase current and the current spectrum, respectively.

TABLE III

Properties of the Four Direct Model Predictive Control SCHEMES

\begin{tabular}{lll}
\hline \hline Methods & Vector slopes & Actuating time \\
\hline C-DMPTC & Fixed and limited & Fixed $\left(=T_{\mathrm{s}}\right)$ \\
DO-DMPTC & Fixed and limited & Unlimited, partially optimized \\
RR-DMPTC & Unlimited and optimized & Unlimited, partially optimized \\
MV-DMPTC & Unlimited and optimized & Unlimited, optimized \\
\hline \hline
\end{tabular}

\section{CONCLUSION}

As a promising nonlinear direct control class, direct model predictive control has already become a viable alternative for both grid-tied active front end and machine side power converter control of wind turbine systems. However, the inherent one-vector-per-control-interval character of the classical DMPTC leads to relatively big control variable ripples, in particular for the two-level cases. This makes the investigation on steady state performance enhanced approaches quite necessary. Following such requirement, the DO-, the RRand the MV-DMPTC solutions have been investigated and discussed comprehensively in this paper. The former two solutions utilize (maximum) two vectors (which can be two active ones or one active and one zero vector), and extend the candidate synthesized vector range to another freedom: phases (directions) other than the fundamental active ones; The latter (MV-DMPTC), by using (maximally) three vectors, makes the whole hexagon plane reachable.

Although the DO-, the RR- and the MV-DMPTC solutions have improved the control performances very considerably, both the required computational efforts and switching frequencies are higher than those for the C-DMPTC solution. However, looking into the future, increasing advanced embedded/real-time hardware (e.g., FPGAs) and the new power electronic devices (e.g., SiC devices) have already been accessible, the computational demands and high switching frequency requirements will not be a problem.

\section{ACKNOWLEDGEMENTS}

This research was made possible by the hardware support of National Instruments and Infineon. Dr.-Ing. Zhenbin Zhang would like to express his gratefulness to Dr.-Ing Martin Schulz (from Infineon); Mr. Marc Backmeyer, Mr. Zwick Wolfgang, Mr. Jan Kniewasser (from NI, Germany) and Mr. Dietmar Schuster (from EAL of TUM) for their help during the test-bench construction.

\section{REFERENCES}

[1] F. Blaabjerg, M. Liserre, and K. Ma, "Power electronics converters for wind turbine systems," IEEE Transactions on Industrial Applications, vol. 48, no. 2, pp. 708-718, 2012.

[2] M. Kazmierkowski, L. Franquelo, J. Rodriguez, M. Perez, and J. Leon, "High-performance motor drives," Industrial Electronics Magazine, IEEE, vol. 5, no. 3, pp. 6-26, Sept. 2011.

[3] J. Rodriguez, M. P. Kazmierkowski, J. R. Espinoza, P. Zanchetta, H. Abu-Rub, H. A. Young, and C. A. Rojas, "State of the art of finite control set model predictive control in power electronics," IEEE Transactions on Industrial Informatics, vol. 9, no. 2, pp. 1003-1016, 2013.

[4] M. Depenbrock, "Direct self-control (DSC) of inverter-fed induction machine," IEEE Transactions on Power Electronics, vol. 3, no. 4, pp. 420-429, 1988.

[5] I. Takahashi and T. Noguchi, "A new quick-response and high-efficiency control strategy of an induction motor," IEEE Transactions on Industrial Applications, vol. IA-22, no. 5, pp. 820-827, 1986.

[6] P. Landsmann and R. Kennel, "Saliency-based sensorless predictive torque control with reduced torque ripple," IEEE Transactions on Power Electronics, vol. 27, no. 10, pp. 4311-4320, Oct. 2012.

[7] Y. Zhang, W. Xie, Z. Li, and Y. Zhang, "Model predictive direct power control of a pwm rectifier with duty cycle optimization," Power Electronics, IEEE Transactions on, vol. 28, no. 11, pp. 5343-5351, Nov. 2013.

[8] H. Fang, Z. Zhang, X. Feng, and R. Kennel, "Ripple-reduced mpdpc for active front end power converters with extended switching vectors and time-optimized control," IET Power Electronics, no. 11, p. 22, Apr. 2016.

[9] Z. Zhang and R. Kennel, "Novel ripple reduced direct model predictive control of three-level NPC active front end with reduced computational effort," in Predictive Control of Electrical Drives and Power Electronics (PRECEDE 2015), Valparaiso, Chile., 2015.

[10] Z. Zhang, H. Fang, F. Gao, J. Rodrguez, and R. Kennel, "Multiplevector model predictive power control for grid-tied wind turbine system with enhanced steady-state control performance," IEEE Transactions on Industrial Electronics, vol. 64, no. 8, pp. 6287-6298, Aug. 2017.

[11] T. Geyer, "Computationally efficient model predictive direct torque control," IEEE Transactions on Power Electronics, vol. 26, no. 10, pp. 2804-2816, 2011.

[12] T. Geyer and D. E. Quevedo, "Multistep direct model predictive control for power electronics -Part 2: Analysis," Energy Convers. Congr. Expo. (ECCE), 2013 IEEE, pp. 1162-1169, 2013.

[13] Z. Zhang, "On control of grid-tied back-to-back power converters and permanent magnet synchronous generator wind turbine systems," Dissertation, Technische Universitt Mnchen, Mnchen, 2016.

[14] Z. Zhang and R. Kennel, "Fully fpga based direct model predictive power control for grid-tied afes with improved performance," in Industrial Electronics Society, IECON 2015 - 41th Annual Conference of the IEEE, Nov. 2015.

[15] J. Z. Zhang, T. Sun, F. Wang, J. Rodriguez, and R. Kennel, “A computationally-efficient quasi-centralized dmpc for back-to-back converter pmsg windturbine systems without dc-link tracking errors," IEEE Transactions on Industrial Electronics, no. 99, p. 1, 2016.

[16] Z. Zhang, H. Xu, M. Xue, Z. Chen, T. Sun, R. Kennel, and C. Hackl, "Predictive control with novel virtual-flux estimation for back-to-back 
power converters," Industrial Electronics, IEEE Transactions on, vol. 62, no. 5, pp. 2823-2834, May 2015.

[17] Z. Zhang, C. Hackl, and R. Kennel, "Fpga based direct model predictive current control of pmsm drives with 31 npc power converter," in PCIM - 2016, Nurnburg, May 2016.

[18] Z. Zhang, H. Xu, M. Xue, Z. Chen, T. Sun, R. Kennel, and C. Hackl, "Predictive control with novel virtual-flux estimation for back-to-back power converters," Industrial Electronics, IEEE Transactions on, vol. 62, no. 5, pp. 2823-2834, May 2015.

[19] Z. Zhang, F. Wang, T. Sun, J. Rodriguez, and R. Kennel, "Fpga based experimental investigation of a quasi-centralized dmpc scheme for a back-to-back converter," Power Electronics, IEEE Transactions on, no. 99 , p. 1, 2015.

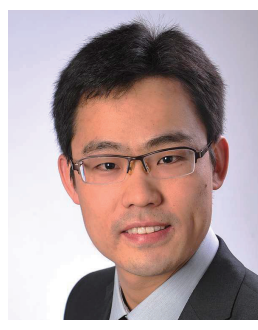

Zhenbin Zhang was born in Shandong, China, in 1984. He received the B.S. degree in electrical engineering in Harbin Engineering University, Harbin, China, in 2008. From 2008 to 2011, he studied in control theory and engineering in Shandong University, Jinan, China. In 2016, he received the Ph.D. degree at the Institute for Electrical Drive Systems and Power Electronics (EAL), Technical University of Munich (TUM), Germany.

Since 2016, he was appointed as the teamleader for "Modern control strategies for electrical drives" group in EAL of TUM. Dr. Zhang has been a Research Fellow of TUM since 2016. His research interests include power electronics, advanced control of electrical drives and power converters, power and renewable energy systems.

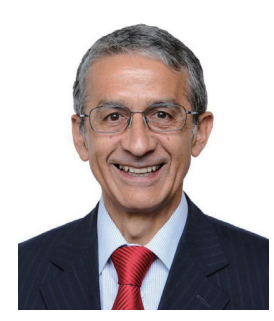

José Rodríguez received the Engineer degree in electrical engineering from the Universidad Federico Santa Maria (UTFSM), Valparaiso, Chile, in 1977 and the Dr.-Ing. degree in electrical engineering from the University of Erlangen, Erlangen, Germany, in 1985. He had been with the Department of Electronics Engineering, University Federico Santa Maria since 1977, where he was a full Professor and the Rector. Currently he is with Universidad Andres Bello, Santiago, Chile and is a full Professor and the Rector. His main research interests include multilevel inverters, new converter topologies, control of power converters, and adjustable-speed drives.

Dr. Rodríguez is Associate Editor of the IEEE Transactions on Power Electronics and IEEE Transactions on Industrial Electronics since 2002. He received the Best Paper Award from the IEEE Industrial Electronics Magazine in 2008, Best Paper Award from the IEEE Transactions on Power Electronics in 2010 and the Best Paper Award from the IEEE Transactions on Industrial Electronics in 2007 and 2011. Dr. Rodríguez is member of the Chilean Academy of Engineering and Fellow of the IEEE.

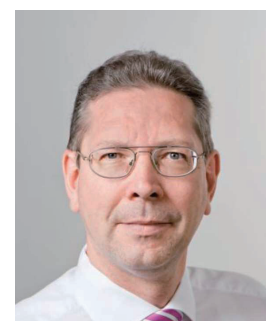

Ralph Kennel was born in 1955 at Kaiserslautern (Germany). In 1979 he got his diploma degree and in 1984 his Dr.-Ing. (Ph.D.) degree from the University of Kaiserslautern. From 1999-2008 he was Professor for Electrical Machines and Drives at Wuppertal University (Germany). Since 2008 he is Professor for Electrical Drive systems and Power Electronics at Technische Universtaet Muenchen (Muenchen, Germany). His main interests today are: Sensorless control of $\mathrm{AC}$ drives, predictive control of power electronics and Hardware-in-the-Loop systems.

Dr. Kennel is a Senior Member of IEEE, a Fellow of IEE and a Chartered Engineer in the UK. Within IEEE he is Treasurer of the Germany Section as well as ECCE Global Partnership Chair of the Power Electronics society (PELS). Dr. Kennel is an Associate Editor of the IEEE Transactions on Power Electronics. 